\title{
CARACTERÍSTICAS DOS PACIENTES COM RESSECAMENTO OCULAR INTERNADOS EM UNIDADE DE TERAPIA INTENSIVA
}

\author{
Danielly Oliveira Pitombeira', Ângela Monic Lima de Souza'1, Ana Paula Nunes de Lima \\ Fernandes², Jéssica Naiara de Medeiros Araújoº ${ }^{2}$ Amanda Barbosa da Silva ${ }^{1}$, Allyne Fortes Vitor ${ }^{3}$
}

\begin{abstract}
RESUMO: Objetivo: identificar características clínicas e sociodemográficas dos pacientes com ressecamento ocular internados em Unidade de Terapia Intensiva. Método: estudo quantitativo, observacional descritivo, realizado em hospital universitário no período de janeiro a junho de 2016, com instrumento composto por dados sociodemográficos e clínicos relacionados ao ressecamento ocular. Após inferência diagnóstica obteve-se amostra de 108 pacientes. Foram realizadas as análises descritivas, medidas de associação e razão de prevalência por meio de pacote estatístico. Resultados: constatou-se predomínio do sexo masculino com 58 (53,7\%) pacientes, idade média 57 anos, 61 (56,6\%) com hipertensão arterial, $84(77,8 \%)$ com fechamento palpebral completo em olho direito e $80(74,1 \%)$ esquerdo, 44 $(40,7 \%)$ com hiperemiano olho direito e 41 (38\%) esquerdo. Teste de Schirmer identificou volumetria insuficiente, mediana de 3 milímetros no olho direito e 4 milímetros no esquerdo. Conclusão: identificou-se informações relevantes para caracterização dos pacientes com ressecamento ocular, predição do fenômeno e consequente assistência qualificada.
\end{abstract}

DESCRITORES: síndromes do olho seco; Saúde ocular; Cuidados críticos; Unidade de terapia intensiva.

\section{PATIENTS WITH DRY EYE ADMITTED TO AN INTENSIVE CARE UNIT}

ABSTRACT: Objective: to identify clinical and sociodemographic characteristics of patients with dry eye admitted to an intensive care unit. Method: observational and descriptive study with a quantitative approach conducted in a university hospital from January to June 2016 through the use of a form with questions on sociodemograpic characteristics and clinical data related to dry eye. After diagnostic inference, a sample of 108 patients was obtained. Descriptive analyzes, association measures and prevalence ratios were performed with the use of a statistical package. Results: there was a prevalence of male patients (53.7\%), mean age of 57 years; 61 (56.6\%) with hypertension; 84 (77.8\%) with complete closure of the eyelid in the right eye and $80(74.1 \%)$ with complete eyelid closure in the left eye; $44(40.7 \%)$ with right eye hyperemia and $41(38 \%)$ with left eye hyperemia. Schirmer test detected a reduced tear volume, median of $3 \mathrm{~mm}$ in the right eye and $4 \mathrm{~mm}$ in the left eye. Conclusion: Relevant information for the characterization of patients with dry eye, prediction of the phenomenon and delivery of proper care was identified.

KEYWORDS: Dry eye syndromes; Eye health; Critical care; Intensive care unit.

\section{PACIENTES CON SEQUEDAD OCULAR INTERNADOS EN UNIDAD DE TERAPIA INTENSIVA}

RESUMEN: Objetivo: identificar características clínicas, sociales y demográficas de los pacientes con sequedad ocular internados en Unidad de Terapia Intensiva. Método: estudio cuantitativo, observacional descriptivo, realizado en hospital universitario en el periodo de enero a junio de 2016, con instrumento compuesto por datos socio demográficos y clínicos asociados a la sequedad ocular. Tras la inferencia diagnóstica, se obtuvo muestra de 108 pacientes. Se realizaron análisis descriptivos, medidas de asociación y razón de prevalencia por medio de paquete estadístico. Resultados: se constató predominio del sexo masculino con 58 (53,7\%) pacientes, edad media 57 años, 61 $(56,6 \%)$ con hipertensión arterial, $84(77,8 \%)$ con cerramiento palpebral completo en ojo derecho y 80 (74, $1 \%)$ izquierdo, 44 (40,7\%) con hiperemia en el ojo derecho y 41 (38\%) izquierdo. Test de Schirmer identificó volumetría insuficiente, mediana de 3 milímetros en el ojo derecho y 4 milímetros en el izquierdo. Conclusión: se identificaron informaciones relevantes para caracterización de los pacientes con sequedad ocular, predicción del fenómeno y consecuente asistencia cualificada.

DESCRIPTORES: Síndrome del ojo seco; Salud ocular; Cuidados críticos; Unidad de terapia intensiva.

${ }^{1}$ Enfermeira. Mestranda no Programa de Pós-Graduação em Enfermagem da Universidade Federal do Rio Grande do Norte. NatalRN-Brasil.

${ }^{2}$ Enfermeira. Mestre em Enfermagem. Doutoranda no Programa de Pós-Graduação em Enfermagem da Universidade Federal do Rio Grande do Norte. Natal-RN-Brasil.

${ }^{3}$ Enfermeira. Doutora em Enfermagem. Docente do Departamento de Enfermagem da Universidade Federal do Rio Grande do Norte. Natal-RN-Brasil. 


\section{INTRODUÇÃO}

Um dos importantes fatores da notoriedade, dada à temática da saúde ocular, deve-se ao enfoque da Organização Mundial da Saúde (OMS) para este assunto. A referida organização elaborou em 2013 o Plano Universal Para a Saúde Ocular, que tem como objetivo reduzir os índices relacionados as doenças oculares entre os anos de 2014-2019. O plano propõe a adoção de estratégias e mudanças nas práticas de saúde para prevenção das deficiências oculares por causas evitáveis por parte das organizações, instituições e profissionais de saúde, tendo em vista que a deficiência visual, principalmente a cegueira, é considerada um problema de saúde pública. Isto porque, segundo dados da OMS,80\% dos casos de cegueira são de causas evitáveis ${ }^{(1)}$.

A síndrome do olho seco, doença que antecede danos à córnea, é definida como uma doença multicausal caracterizada pela instabilidade do filme lacrimal, a partir de alterações na função de um ou mais elementos que compõe uma unidade funcional da lágrima e,quando não reabilitada, gera comprometimento funcional irreversível ${ }^{(2)}$.

O ressecamento ocular por sua vez, é uma resposta humana as alterações considerada um atributo crítico que define o olho seco e é inferido por meio de um teste de volumetria do filme lacrimal positivo, associado a um sinal e/ou sintoma ocular ${ }^{(3)}$.

No contexto da Unidade de Terapia Intensiva (UTI),a atenção à saúde está direcionada as funções sistêmicas consideradas essenciais para manutenção da vida. Em decorrência desta priorização, o cuidado ocular muitas vezes deixa de ser prestado, o que gera complicações ao longo da internação. Estudos sobre a temática constataram que os fatores de risco predisponentes ao aparecimento de lesões oculares (fenômeno que sucede a síndrome do olho seco) encontrados na UTI, foram, dentre outros, o uso de suporte ventilatório invasivo, bloqueadores musculares, edema e sedação.Além disso, as chances variam de acordo com o tempo de internação, uso de dispositivos ventilatórios invasivos e uso de drogas ${ }^{(4)}$.

Nesse sentido, percebe-se a importância de realizar uma promoção ao cuidado ocular em pacientes internados em UTI, pois apesar de ser um cuidado incipiente, a ausência deste acarretará danos permanentes capazes de interferir na qualidade de vida do indivíduo ${ }^{(5)}$.

Diante da problemática levantada, estabeleceu-se a seguinte questão de pesquisa: quais as características clínicas e sociodemográficas dos pacientes com ressecamento ocular internados em unidades de terapia intensiva?

Este estudo justifica-se pela importância de identificar as características clínicas e sociodemográficas de pacientes internados na UTI, como forma de verificar a ocorrência das variáveis nos pacientes com ressecamento ocular.

Desta forma, objetivou identificar características clínicas e sociodemográficas dos pacientes com ressecamento ocular internados em Unidade de Terapia Intensiva. Está de acordo com o preconizado pela OMS em seu Plano Universal e pretende contribuir positivamente com a temática, fornecendo informações que sirvam de subsídio para as ações em saúde e que possam contribuir com a diminuição da prevalência desta condição.

\section{METODOLOGIA}

Trata-se de um estudo de abordagem quantitativa do tipo observacional descritivo, realizado em um Hospital Universitário de referência, localizado no município de Natal, Rio Grande do Norte, Brasil.

Os participantes do estudo encontravam-se internados na Unidade de Terapia Intensiva adulta e obedeceram aos seguintes critérios de inclusão: estarem internados na UTI do referido hospital, com tempo de internação superior a 24 horas; idade igual ou superior a 18 anos; não possuírem danos que envolvam a lubrificação ocular ou estarem em uso de tratamentos oculares no momento da coleta de dados; e apresentarem diagnóstico de ressecamento ocular obtido através da inferência diagnóstica, por dupla de especialistas. Foram excluídos pacientes com agitação ou em situações de emergência durante a coleta. 
A fim de reduzir viés de aferição, foi realizado antes do início da coleta de dados, um treinamento de caráter teórico/prático sobre o objeto de estudo e as técnicas de mensuração das variáveis, para calibrar os entrevistadores. Posterior ao treinamento, foi realizada uma avaliação teórica com correção cegada e apenas os entrevistadores que obtiveram nota igual ou superior a 7,0 foram considerados aptos a coletar.

A coleta de dados ocorreu de janeiro a junho de 2016 por meio de entrevista estruturada. Utilizouse como instrumento um formulário composto por dados clínicos e sociodemográficos. Como variáveis relacionadas aos dados sociodemográficos, foram elencadas: sexo, idade, estado civil, local de nascimento, local que reside, grau de escolaridade, ocupação, renda familiar mensal, dependentes da renda familiar e praticantes ou não de alguma religião. As variáveis clínicas foram: tipo e motivo da internação na UTI, tipo de pós-operatório, setor de procedência e comorbidades.

Foi realizada a avaliação ocular nos pacientes selecionados em um único momento, com as seguintes variáveis: exposição do globo ocular, hiperemia, secreção mucosa, edema palpebral, edema conjuntival (quemose), hemorragia conjuntival, proptose e teste de Schirmer.

O teste de Schirmer é utilizado para diagnosticar o ressecamento ocular a partir da volumetria lacrimal. O teste é realizado com fitas de papel filtro padrão de $5 \times 35$ milímetros, com os cinco primeiros milímetros encurvados. A parte encurvada é colocada na porção externa da pálpebra inferior, e permanece por 5 minutos no paciente com os olhos fechados. Decorridos cinco minutos, a fita é retirada e avaliada pela quantidade de milímetros umedecidos no filtro. O teste é considerado negativo (normal) quando a medida for maior ou igual a 10milímetros ${ }^{(6)}$.

Ressalta-se que este estudo faz parte de uma investigação maior cuja amostra foi obtida por meio do cálculo de populações finitas $n=Z_{\alpha}{ }^{2} \cdot p \cdot q \cdot N /\left[Z_{\alpha}{ }^{2} \cdot p \cdot q+(N-1) \cdot e^{2}\right]$, que resultou em 206 pacientes. Este quantitativo foi submetido a inferência diagnóstica, de acordo com as variáveis de avaliação ocular. A inferência foi realizada por uma dupla de especialistas integrantes do Núcleo de Estudos e Pesquisa em Enfermagem Clínica (NEPEC) da Universidade Federal do Rio Grande do Norte, as convergências foram resolvidas por consenso, que determinou uma amostra de 108 pacientes com ressecamento ocular.

Como critérios para a detecção da condição de ressecamento ocular, foram considerados os pacientes com um teste de volumetria positivo associado a um sinal e/ou sintoma ocular, que incluem desconforto e/ou distúrbios visuais ${ }^{(3)}$.

Os dados obtidos foram tabulados com dupla entrada e exportados para um pacote de dados estatísticos para a realização da análise. Para a análise descritiva, foram consideradas as frequências, medidas do centro da distribuição e suas variabilidades. Para verificar a normalidade dos dados, foram observadas a assimetria, curtose e os critérios para normalidade ${ }^{(7)}$ bem como o teste de Shapiro-Wilk. Para medidas associativas dos dados categóricos nominais foram utilizados o teste Qui-quadrado de Pearson e quando as frequências esperadas foram menores que cinco foi aplicado o teste de Fisher. A Razão de Prevalência foi utilizada para verificar a magnitude da associação.

Antes de ser iniciada a coleta de dados, foi solicitado a assinatura do Termo de Consentimento Livre e Esclarecido (TCLE) com duas cópias, uma arquivada com o participante quando consciente e orientado ou entregue ao acompanhante responsável e outra com a coordenadora da pesquisa. Além disso, foi garantido sigilo dos dados obtidos e sua utilização para pesquisas científicas. Nesse sentido, o presente estudo obedeceu aos princípios éticos da resolução no 466/2012 do Conselho Nacional de Saúde (CNS) ${ }^{(8)}$ e obteve parecer favorável do CEP sob número 918.510.

\section{RESUltAdOS}

Os dados sociodemográficos da amostra estão representados na Tabela 1. Constatou-se que dos 108 pacientes que apresentaram o ressecamento ocular, 58 (53,7\%) eram do sexo masculino, 63 (62,4\%) com companheiro, $71(71 \%)$ naturais do interior do estado, $38(41,3 \%)$ com ensino fundamental incompleto e $43(45,7 \%)$ são aposentados. Referente à idade, obteve-se uma média de 57,7 anos e renda familiar mediana de $\mathrm{R} \$ 1.760,00$. 
Tabela 1 - Caracterização sociodemográfica dos pacientes com ressecamento ocular na Unidade de Terapia Intensiva. Natal, RN, Brasil, 2016

\begin{tabular}{|c|c|c|c|c|c|c|}
\hline \multicolumn{3}{|c|}{ Variáveis } & \multicolumn{2}{|r|}{$n$} & \multicolumn{2}{|r|}{$\%$} \\
\hline \multicolumn{7}{|l|}{ Sexo } \\
\hline \multicolumn{3}{|l|}{ Masculino } & \multicolumn{2}{|r|}{58} & \multicolumn{2}{|r|}{53,7} \\
\hline Feminino & & & \multicolumn{2}{|r|}{50} & \multicolumn{2}{|r|}{46,3} \\
\hline \multicolumn{7}{|l|}{ Estado civil** } \\
\hline \multirow{2}{*}{\multicolumn{3}{|c|}{$\begin{array}{l}\text { Com companheiro } \\
\text { Sem companheiro }\end{array}$}} & \multicolumn{2}{|r|}{63} & \multicolumn{2}{|r|}{62,4} \\
\hline & & & & 38 & \multicolumn{2}{|r|}{37,6} \\
\hline \multicolumn{7}{|l|}{ Local de nascimento** } \\
\hline \multicolumn{3}{|l|}{ Interior do RN } & \multicolumn{2}{|r|}{71} & \multicolumn{2}{|r|}{71} \\
\hline \multirow{2}{*}{\multicolumn{3}{|c|}{$\begin{array}{l}\text { Capital do RN } \\
\text { Outros }\end{array}$}} & \multirow{2}{*}{\multicolumn{2}{|c|}{$\begin{array}{l}20 \\
09\end{array}$}} & \multicolumn{2}{|r|}{20} \\
\hline & & & & & \multicolumn{2}{|r|}{9} \\
\hline \multicolumn{7}{|l|}{ Local onde reside** } \\
\hline \multirow{2}{*}{\multicolumn{3}{|c|}{$\begin{array}{l}\text { Interior do RN } \\
\text { Capital do RN }\end{array}$}} & \multicolumn{2}{|r|}{55} & \multicolumn{2}{|r|}{54,4} \\
\hline & & & \multirow{2}{*}{\multicolumn{2}{|c|}{$\begin{array}{l}42 \\
04\end{array}$}} & & 41,6 \\
\hline Outros & & & & & & 4 \\
\hline Grau de escolaridade** & & & & & & \\
\hline Ensino fundamental incomple & & & & 38 & & 41,3 \\
\hline Ensino fundamental completo & & & & 18 & & 19,6 \\
\hline Ensino médio completo & & & & 12 & & 13 \\
\hline Analfabeto & & & & 12 & & 13 \\
\hline Superior completo & & & & 04 & & 4,3 \\
\hline Superior incompleto & & & & 03 & & 3,3 \\
\hline Não sabe informar & & & & 03 & & 3,3 \\
\hline Ensino médio incompleto & & & & 02 & & 2,2 \\
\hline Ocupação** & & & & & & \\
\hline Aposentado & & & & 43 & & 45,7 \\
\hline Atividade remunerada & & & & 32 & & 34 \\
\hline Atividade sem remuneração & & & & 09 & & 9,6 \\
\hline Licença saúde & & & & 05 & & 5,3 \\
\hline Estudante & & & & 03 & & 3,2 \\
\hline Sem atividade & & & & 02 & & 2,1 \\
\hline Religião** & & & & & & \\
\hline Praticante & & & & 82 & & 88,2 \\
\hline Não praticante & & & & 11 & & 11,8 \\
\hline & Média & Desvio padrão & Mediana & Máximo & Mínimo & Valor de $\mathrm{p}^{*}$ \\
\hline Idade & 57,73 & 14,552 & 58 & 96 & 19 & 0,419 \\
\hline Renda familiar*** & $2.057,1$ & $1.717,3$ & 1760 & 8880 & 0 & $<0,001$ \\
\hline № de dependentes familiares & 3,31 & 1,622 & 3 & 9 & 0 & $<0,001$ \\
\hline
\end{tabular}

*Legenda: *Teste Shapiro-Wilk; ${ }^{* *}$ Dados ausentes; ${ }^{* * *}$ Renda familiar em reais. 
No tocante às variáveis clínicas (Tabela 2), destacam-se na amostra as internações por cirurgias eletivas com 55 (50,9\%) pacientes, motivo de internação por pós-operatório com 54 (50\%) pacientes e tempo de internação de mediana de 1 dia.

Tabela 2 - Caracterização clínica dos tipos de internação em Unidade de Terapia Intensiva.Natal, RN, Brasil, 2016.

\begin{tabular}{|c|c|c|c|c|}
\hline Variáveis & & $\mathbf{n}$ & & $\%$ \\
\hline \multicolumn{5}{|l|}{ Tipo de internação } \\
\hline \multicolumn{2}{|l|}{ Cirurgia eletiva } & 55 & & 50,9 \\
\hline \multicolumn{2}{|l|}{ Cirúrgica de urgência /emergência } & 28 & & 25,9 \\
\hline \multicolumn{2}{|l|}{ Clínica } & 25 & & 23,2 \\
\hline \multicolumn{2}{|l|}{ TOTAL } & 108 & & 100 \\
\hline \multicolumn{5}{|l|}{ Motivo de internação } \\
\hline \multicolumn{2}{|l|}{ Pós-operatório } & 54 & & 50 \\
\hline \multicolumn{2}{|l|}{ Distúrbio cardíaco } & 25 & & 23,1 \\
\hline \multicolumn{2}{|l|}{ Choque } & 14 & & 13 \\
\hline \multicolumn{2}{|l|}{ Distúrbio pulmonar } & 12 & & 11,1 \\
\hline \multicolumn{2}{|l|}{ Distúrbio neurológico } & 07 & & 6,5 \\
\hline \multicolumn{2}{|l|}{ Distúrbio infeccioso } & 06 & & 5,6 \\
\hline \multicolumn{2}{|l|}{ Distúrbio gastrointestinal } & 06 & & 5,6 \\
\hline \multicolumn{2}{|l|}{ PCR } & 05 & & 4,6 \\
\hline \multicolumn{2}{|l|}{ Distúrbio renal } & 04 & & 3,7 \\
\hline \multicolumn{2}{|l|}{ Encefalopatia hepática } & 03 & & 2,8 \\
\hline \multicolumn{2}{|l|}{ Distúrbio eletrolítico } & 02 & & 1,9 \\
\hline \multicolumn{2}{|l|}{ Distúrbio vascular } & 01 & & 0,9 \\
\hline \multicolumn{2}{|l|}{ Distúrbio metabólico } & 01 & & 0,9 \\
\hline \multicolumn{2}{|l|}{ Neoplasia } & 01 & & 0,9 \\
\hline \multicolumn{2}{|l|}{ Síndrome HELLP } & 01 & & 0,9 \\
\hline \multicolumn{5}{|l|}{ Tipo de pós-operatório } \\
\hline \multicolumn{2}{|l|}{ Não se aplica } & 53 & & 49,1 \\
\hline \multicolumn{2}{|l|}{ Cirurgia abdominal } & 19 & & 17,6 \\
\hline \multicolumn{2}{|l|}{ Cirurgia cardíaca } & 13 & & 12 \\
\hline \multicolumn{2}{|l|}{ Angioplastia coronariana } & 12 & & 11,1 \\
\hline Cirurgia neurológica & & 04 & & 3,7 \\
\hline Procedimentos invasivos (colocação de cateteres) & & 03 & & 2,8 \\
\hline Cirurgia torácica & & 02 & & 1,9 \\
\hline Cirurgia renal & & 01 & & 0,9 \\
\hline Cirurgia vascular & & 01 & & 0,9 \\
\hline Setor de procedência** & & & & \\
\hline Centro-cirúrgico & & 36 & & 33,3 \\
\hline Unidade de clínica médica & & 31 & & 29 \\
\hline Hemodinâmica & & 21 & & 19,6 \\
\hline Outro hospital & & 14 & & 13,1 \\
\hline Unidade de clínica cirúrgica & & 04 & & 3,7 \\
\hline Ambulatório & & 01 & & 0,9 \\
\hline Desvio padrão & Mediana & Mínimo & Máximo & Valor $\mathrm{p}^{*}$ \\
\hline $\begin{array}{l}\text { Dias de } \\
\text { internação }\end{array}$ & 1,00 & 1,00 & 26,00 & $<0,001$ \\
\hline
\end{tabular}


Os dados levantados sobre as comorbidades (Tabela 3) revelaram que 61 (56,6\%) pacientes da amostra eram portadores de hipertensão arterial sistêmica, seguido de diabetes mellitus, em 29 (26,9\%) pacientes e neoplasias, em 28 (25,9\%) pacientes.

Tabela 3 - Comorbidades gerais apresentadas pelos pacientes com ressecamento ocular internados em Unidade de Terapia Intensiva. Natal, RN, Brasil, 2016

\begin{tabular}{lcc}
\hline & Variáveis & N \\
\hline Hipertensão arterial sistêmica & 61 & 56,6 \\
Diabetes mellitus & 29 & 26,9 \\
Neoplasias & 28 & 25,9 \\
Insuficiência renal crônica & 23 & 21,3 \\
Coronariopatias & 15 & 13,9 \\
Insuficiência cardíaca congestiva & 12 & 11,1 \\
Respiratórias & 11 & 10,2 \\
Dislipidemias & 8 & 7,4 \\
Acidente vascular encefálico & 7 & 6,5 \\
Hepatopatias & 7 & 6,5 \\
Doenças vasculares & 6 & 5,6 \\
Arritmias & 5 & 4,6 \\
Obesidade & 4 & 3,7 \\
Doença autoimune & 4 & 3,7 \\
Doenças infecciosas & 4 & 3,7 \\
Doenças das valvas cardíacas & 3 & 2,8 \\
Doença na tireoide & 3 & 2,8 \\
Gota & 2 & 1,9 \\
Catarata & 2 & 1,9 \\
Hiperplasia prostática benigna & 2 & 1,9 \\
Doenças neurológicas & 1 & 0,9 \\
Doenças hematológicas & 1 & 0,9 \\
Pós-operatório tardio & 1 & 0,9 \\
Transplante renal & 1 & 0,9 \\
\hline
\end{tabular}

Sobre as variáveis da avaliação ocular,a maioria dos pacientes apresentaram fechamento palpebral completo do olho esquerdo e direito, quando em relação à exposição do globo ocular.A hiperemia (vermelhidão na conjuntiva) refere-se a presença de vasos sanguíneos dilatados, classificados de acordo com sua extensão, número de vasos dilatados e área hiperemiada. A hiperemia superficial foi vista em $44(40,7 \%)$ olhos direitos e 41 (38\%) olhos esquerdos e outra quantidade significativa apresentou características de hiperemia mais profunda (Tabela 4).

Tabela 4 - Caracterização dos sinais e dados clínicos e resultado do Teste de Schirmer nos pacientes com ressecamento ocular internados em Unidade de Terapia Intensiva. Natal, RN, Brasil, 2016. (continua)

\begin{tabular}{lcccc}
\multicolumn{1}{c}{ Variáveis } & $\mathbf{N}$ & $\mathbf{n}$ & $\%$ & $\%$ \\
\hline \multicolumn{1}{c}{ Avaliação ocular - sinais clínicos } & OD & OE & OD & OE \\
\hline Exposição do globo ocular & & & & \\
Fechamento palpebral completo & 84 & 80 & 77,8 & 74,1 \\
$1 / 3$ inferior da córnea exposta & 10 & 13 & 9,3 & 12 \\
$1 / 2$ da córnea exposta & 7 & 4 & 6,5 & 3,7 \\
Conjuntiva exposta & 7 & 6 & 6,5 & 5,6 \\
Córnea completamente exposta & 0 & 5 & 0 & 4,6 \\
\hline
\end{tabular}




\begin{tabular}{|c|c|c|c|c|c|c|c|c|}
\hline \multicolumn{5}{|c|}{ Hiperemia ocular } & 44 & 41 & 40,7 & 38 \\
\hline \multicolumn{5}{|c|}{ Hiperemia superficial } & 22 & 27 & 20,4 & 25 \\
\hline \multicolumn{5}{|c|}{ Vasos sanguíneos dilatados em região conjuntival perilimbar } & 9 & 6 & 8,3 & 5,6 \\
\hline \multirow{2}{*}{\multicolumn{5}{|c|}{$\begin{array}{l}\text { Vasos sanguíneos numerosos e obviamente dilatados em conjuntiva e início da } \\
\text { córnea }\end{array}$}} & 8 & 8 & 7,4 & 7,4 \\
\hline & & & & & 25 & 26 & 23,1 & 24,1 \\
\hline \multicolumn{9}{|c|}{$\begin{array}{l}\text { Vasos sanguíneos numerosos e dilatados dispersos por toda superfície ocular } \\
\text { Não se aplica }\end{array}$} \\
\hline \multicolumn{5}{|l|}{ Quemose } & 39 & 37 & 36,1 & 34,3 \\
\hline \multicolumn{5}{|c|}{ Edema palpebral } & 34 & 31 & 31,5 & 28,7 \\
\hline \multicolumn{5}{|l|}{ Lagoftalmia } & 25 & 24 & 23,1 & 22,2 \\
\hline \multicolumn{5}{|c|}{ Hemorragia conjuntival } & 05 & 04 & 4,6 & 3,7 \\
\hline \multicolumn{5}{|l|}{ Proptose } & 05 & 03 & 4,6 & 2,8 \\
\hline Variáveis & Desvio padrão & Mediana & Mínimo & Máximo & \multicolumn{4}{|c|}{ Valor $\mathbf{p}^{*}$} \\
\hline Schirmer OD & 5,17 & 3 & 1 & 25 & \multicolumn{4}{|c|}{$p<0,001$} \\
\hline Schirmer OE & 4,29 & 4 & 1 & 23 & & $\mathrm{p}<$ & & \\
\hline
\end{tabular}

Legenda: OD: olho direito; OE: olho esquerdo

\section{DISCUSSÃO}

As cirurgias abdominais foram o tipo de pós-operatório mais prevalente no presente estudo, o que induz ao levantamento da hipótese de que a cirurgia é um fator predisponente a ocorrência do ressecamento ocular por dois principais motivos: primeiro por favorecer a perda de volume que resulta em uma diminuição da produção do filme lacrimal, e segundo por utilizar anestésicos do tipo geral.

Os anestésicos gerais causam depressão do sistema nervoso central, em consequência disto ocorre inibição dos reflexos motores e sensoriais ${ }^{(9)}$. Com a inibição desses reflexos ocorre uma diminuição do reflexo de piscar e da produção reflexa lacrimal. O que impede o fechamento palpebral completo ${ }^{(10)}$. Com base em tais constatações, acredita-se que o paciente se torne predisposto a apresentar o ressecamento ocular a partir do procedimento cirúrgico, por apresentar esse conjunto de fatores: perda de volume relacionado ao procedimento cirúrgico, utilização de anestésicos, perda do reflexo de piscar e da produção lacrimal reflexa, o que ocasiona assim a diminuição da produção e aumento da evaporação do filme lacrimal.

Sobre o número de dias de internação um estudo recente mostrou que o tempo de internação em UTI é importante para o aparecimento de lesões oculares devido à exposição prolongada aos fatores de risco deste ambiente. Por serem pacientes críticos que necessitam de assistência especializada, os cuidados considerados básicos como o cuidado ocular acabam sendo negligenciados, além disso, estão expostos a fatores como uso ventilação mecânica, sedativos, múltiplas medicações e ar condicionado(10-11).

Dentre as comorbidades investigadas a hipertensão arterial sistêmica foi a mais apresentada pelos pacientes do estudo. Neste contexto, a literatura constata o fato de que o regime de tratamento com o uso de inibidores da enzima conversora da angiotensina podem influenciar no surgimento do ressecamento ocular por interferir na eficiência do filme lacrimal ${ }^{(12)}$.

Em relação à avaliação ocular, houve discreta diferença entre OD e OE quanto ao fechamento palpebral completo. A diminuição da frequência do piscar e o fechamento palpebral incompleto podem comprometer a integridade da superfície ocular. A lagoftalmia em conjunto com a diminuição do reflexo do piscar colabora para um processo de evaporação da lágrima de forma mais rápida, o que leva a redução da lubrificação da superfície ocular e limita de forma significativa a remoção dos microorganismos da superfície ocular ${ }^{(13)}$.

Os dados coletados nesta pesquisa revelam que houve uma grande prevalência de hiperemia nos pacientes, sobretudo quando somadas todas as classificações. Estimou-se que $76,8 \%$ apresentaram hiperemia no OD e $76 \%$ no OE. A hiperemia é um sinal resultante da presença de vasos sanguíneos dilatados na conjuntiva, caracterizada por vermelhidão na conjuntiva ocular e causada pelo mau 
funcionamento das estruturas especializadas para a manutenção da lubrificação na superfície ocular ${ }^{(3)}$.

Os dados pesquisados mostraram que $36,1 \%$ dos pacientes apresentaram edema na conjuntiva (quemose) no OD e $34,3 \%$ no OE. Esses dados obtidos condizem com dados de um outro estudo encontrado na literatura, com pacientes de características similares, os quais apontaram que a quemose foi encontrado em $37,07 \%$ dos pacientes pesquisados ${ }^{(14)}$.

Pode-se entender o edema palpebral como o acúmulo de líquidos na região palpebral que pode levar ao comprometimento do piscar e ao fechamento palpebral incompleto. Somado a isso, a pesquisa obteve como resultado considerável para edema palpebral, com 31,5\% no OD e $28,7 \%$ no OE dos pacientes, além de uma associação sugerida pela estatística inferencial do teste para ambos os olhos.

O teste de Schirmer obteve valores de mediana de 3,00 para OD e 4,00 para OE, o que constata a presença do ressecamento ocular, pois a volumetria insuficiente é um atributo que define a presença do fenômeno. Visto que o teste de volumetria pode indicar redução da produção da lágrima pela hipossecreção da glândula lacrimal, como pelo aumento da evaporação, os quais provocam instabilidade do filme lacrimal e posteriormente o ressecamento ocular quando associado a pelo menos um sinal ou sintoma clínico ${ }^{(3)}$.

O estudo apresentou como limitações o fato de ter sido realizado em uma única Unidade de Terapia Intensiva e, portanto, seus resultados podem não representar a população em geral. Além disso, por se tratar de um estudo observacional transversal, não foi possível estabelecer relações causais entre a exposição às variáveis e a ocorrência do ressecamento ocular.

\section{CONCLUSÃO}

Com o desenvolvimento desta pesquisa, foi possível observar a vulnerabilidade aos danos oculares dos pacientes internados na Unidade de Terapia Intensiva do hospital estudado. É importante que os profissionais de enfermagem, responsáveis pelo cuidado contínuo a este público, garantam ações voltadas a saúde dos olhos, a fim de evitar danos que possam comprometer a qualidade de vida destes pacientes.

O conhecimento acerca das características clínicas e sociodemográficas dos pacientes com ressecamento ocular possui estimada relevância, por permitir ao enfermeiro o diagnóstico correto, que possibilite planejamento adequado das ações de enfermagem, direcionadas à prevenção e controle de possíveis agravos à saúde ocular.

Estudos observacionais são capazes de caracterizar uma população e determinar fatores responsáveis pela ocorrência de uma doença. Enquadra-se como importante ferramenta para subsidiar no planejamento e adoção de estratégias capazes de prevenir os agravos em saúde e pode sugerir importantes hipóteses a serem testadas. Entretanto, apresentam suas limitações no que se refere à coleta dos dados, por permitir somente os dados observados num recorte único de tempo e por ser incapazes de inferir causa e efeito.

Os resultados obtidos permitiram levantar hipóteses e possibilidades de associações entre as variáveis, porém é imprescindível que sejam testadas por meio de outros delineamentos de pesquisa no futuro.

\section{AGRADECIMENTOS}

Ao Conselho Nacional de Desenvolvimento Científico e Tecnológico - CNPq, por financiar a presente pesquisa, por meio da chamada pública MCTI/CNPQ/Universal-14/2014 (processo n. 444290/2014-1). 


\section{REFERÊNCIAS}

1. Organização Mundial da Saúde (OMS). Salud ocular universal: unplan de acción mundial para 2014-2019. [Internet] España: OMS; 2013 [acesso em 29 set 2016]. Disponível: http://www.who.int/blindness/AP2014_19_Spanish.pdf.

2. Holland EJ, Lee WB, Mannis MJ. Doenças da Superfície Ocular:Córnea, conjuntiva e filme lacrimal. Rio de Janeiro: Elsevier; 2015.

3. Craig JP, Nichols KK, Akpek EK, Caffery B, Dua HS, Joo CK, et al. TFOS DEWS II Definition and Classification Report. The Ocular Surface. [Internet] 2017;5(3) [acesso em 12 dez 2017]. Disponível: https://doi.org/10.1016/j. jtos.2017.05.008.

4. Oliveira RS, Fernandes APNL, Botarelli FR, Araújo JNM, Barreto VP, Vitor AF. Risk factors for injury in the cornea in critical patients in intensive care: an integrative review. J. res.: fundam. care online. [Internet] 2016;8(2) [acesso em 29 out 2016]. Disponível: http://dx.doi.org/ 10.9789/2175-5361.2016.v8i2.4423-4434.

5. de Araújo DD, Almeida NG, Silva PMA, Ribeiro NS, Werli-Alvarenga A, Chianca TCM. Prediction of risk and incidence of dry eye in critical patients. Rev. Latino-Am. Enfermagem. [Internet] 2016;24 [acesso em 28 out 2016]. Disponível: http://dx.doi.org/10.1590/1518-8345.0897.2689.

6. Alves JS. Olho seco: uma abordagem didática. Rio de Janeiro: E-papers; 2010.

7. Martínez-González MA, organizador. Bioestadística amigable. 3ª ed. Espanã: Elsevier; 2014.

8. Conselho Nacional de Saúde.Resolução n. 466, de 12 dezembro 2012. Dispõe sobre Diretrizes e normas regulamentadores de pesquisas envolvendo seres humanos. Brasília: Conselho de Saúde; 2012.

9. Nicolaou N, Hourris S, Alexandrou P, Georgiou J. EEG-Based Automatic Classification of 'Awake' versus 'Anesthetized' State in General Anesthesia Using Granger Causality. PLoS one. [Internet] 2012;7(3) [acesso em 30 set 2016]. Disponível: https://doi.org/10.1371/journal.pone.0033869.

10. Demirel S, Cumurcu T, Firat P, Aydogan MS, Doganay S. Effective management of exposure keratopathy developed in intensive care units: The impact of an evidence based eye care education programme. Intensive and Critical Care Nursing. [Internet] 2014;30(1) [acesso em 02 set 2016]. Disponível: http://dx.doi.org/10.1016/j.iccn.2013.08.001.

11. Câmara VGN, Araújo JNM, Fernandes APNL, Botarelli FR, da Silva AB, Medeiros RAC, et al. Methods for Detection of Dry Eye in Critically III Patients: an Integrative Review. Inter. Arch. of Med. [Internet] 2016;9(58) [acesso em 02 set 2016]. Disponível: http://dx.doi.org/10.3823/1929.

12. Herdman TH, Kamitsuru S, editores. NANDA International Nursing Diagnoses: Definitions and Classification 20152017. Oxford: Wiley Blackwell; 2014.

13. Grixti A, Sadri M, Edgar J, Datta AV. Common ocular surface disorders in patients in intensive care units. The Ocular Surface. [Internet] 2012;10(1) [acesso em 02 set 2016]. Disponível: http://dx.doi.org/10.1016/j.jtos.2011.10.001.

14. Araújo DD. Predição de risco e incidência de olho seco em pacientes críticos [dissertação]. Belo Horizonte (MG): Universidade Federal de Minas Gerais; 2014. Disponível: http://www.bibliot=1.ecadigital.ufmg.br/dspace/bitstream/ handle/1843/ANDO-9RGEEK/diego_dias_de_ara_jo.pdf?sequence. 